\title{
Synchronous Acquisition and Analysis of Ultrasonic Spectral Information for the Characterization of Particle Size Distribution
}

\author{
Nan Jia, ${ }^{1}$ Jianfei Gu, ${ }^{2}$ Huinan Yang, ${ }^{1}$ and Mingxu Su $\mathbb{D}^{1}$ \\ ${ }^{1}$ Key Laboratory of Shanghai for Power Engineering Multi-Phase and Heat Transfer, University of Shanghai for Science \\ and Technology, Shanghai 200093, China \\ ${ }^{2}$ School of Electrical and Automatic Engineering, Changshu Institute of Technology, Changshu 215500, China
}

Correspondence should be addressed to Mingxu Su; sumx@usst.edu.cn

Received 2 December 2018; Revised 27 February 2019; Accepted 12 March 2019; Published 2 May 2019

Academic Editor: Andrea Cusano

Copyright (C) 2019 Nan Jia et al. This is an open access article distributed under the Creative Commons Attribution License, which permits unrestricted use, distribution, and reproduction in any medium, provided the original work is properly cited.

Particle sizing methods have gained considerable attention in the past few decades, but there is still a big challenge in high concentration situations (i.e., volume fraction $>10 \%$ ). However, the ultrasonic spectroscopy technique is a common tool for the noninvasive determination of essential parameters for high concentration systems by analyzing ultrasonic spectra with inversion algorithms, including the particle size distribution (PSD), volume fraction of each phase, and physicochemical properties. For the ultrasonic measurements, proper acquisition and analysis of ultrasonic spectra are becoming significant in order to understand the relationship between the unknown parameters and the ultrasonic spectra. In the work, an experimental setup was provided to synchronously acquire ultrasonic reflection and transmission signals. A series of experiments were performed on silicon-water solutions at volume fractions $8 \%, 10 \%$, and $12 \%$ to obtain the ultrasonic attenuation spectra and ultrasonic phase velocity spectra based on different measurement methods, i.e., the pulse-echo method, reference reflection method, and through-transmission method, respectively. Based on the Epstein-Carhart-Allegra-Hawley (ECAH) forward model, genetic algorithm (GA) and optimum regularization technique (ORT) algorithms were implemented to determine PSD with the measured spectra; the obtained PSD was then compared with the optical microscope method. It revealed that the spectra obtained by different measurement methods showed individual features while the obtained PSD was consistent and the volume median diameters were within a deviation of $10 \%$ with GA and ORT algorithms. The differences and characteristics of these three measurement methods for signal acquisition and interpretation were discussed and presented to provide an evaluation and recommendation for ultrasonic particle sizing.

\section{Introduction}

With the rapid development of the industrial processes for chemical, material, and pharmaceutical productions and food processing, particle sizing technology has obtained considerable concerns and investigations [1,2]. In various dispersed systems, the particle size obviously influences the material properties such as stability, appearance, and performance. For instance, the tinctorial power is usually dominated by the pigment size, the additive size significantly affects the film strength and wear resistance, and the catalyst size and its distribution also influence the catalytic activity $[3,4]$. Therefore, it is useful and meaningful to measure particle size in various industrial fields. The most widely employed measurement techniques for particle size distribution (PSD) are based on static or dynamic light scattering and image analysis [5]. However, compared with these competitive approaches, the method of ultrasonic spectroscopy also possesses the remarkable advantages in the highly concentrated suspension and even in the optical opaque medium, due to the strong penetrability of ultrasonic wave propagating through the medium [6-9]. Meanwhile, this approach can also permit the noninvasive and nondestructive measurements to avoid contaminating samples as well as allow measurements under circumstances close to process conditions [10, 11]. Furthermore, this method is also characterized by commercially available and endurable experimental setups, so it is right these characteristics that promote the ultrasonic spectroscopy 
technique development and application, for example, measuring the concentration and size of the pulverized coal system [12] and monitoring the characteristics of the dynamic crystallization process [13] and the process tomography for gas-solid two-phase flow characterization [14].

It is a truth universally acknowledged that ultrasound is an important carrier to reflect the size and distribution of particles in suspensions. The overall process for particle size distribution of a suspension measured using ultrasonic spectra can be focused on two aspects: one is the adequate measurement of frequency-dependent ultrasonic spectra, and the other is the appropriate implementation using the ultrasonic theoretical model and inversion algorithm [15, 16]. It is essential to carry out both steps carefully for an accurate measurement.

On one hand, as far as the measurement itself is concerned, it is crucial to take extreme care in guaranteeing the device well-designed (e.g., a collimated beam and a smooth interface of buffer rods) in order to acquire the reliable ultrasonic spectra. In principle, it allows for in situ process measurements, given that ultrasonic wave information can be obtained in a fast and nondestructive way. The available approaches for particle size distribution based on the ultrasonic spectrum technique are mainly classified into the ultrasonic attenuation method and ultrasonic phase velocity method [17, 18]. And both spectra are measured after an ultrasonic wave transmitting through the suspension with dispersed particles and can provide the similar information such as the particle size and its distribution. But the sensitivity and information contents of experimental data are different since the attenuation spectrum is related to the ultrasonic amplitude while the phase velocity is related to the phase shift and time difference. Furthermore, different spectral analysis methods have a very good complementarity if their characteristics can be fully analyzed and utilized; for example, temperature is particularly essential for accurate phase velocity but is generally less critical where the ultrasonic attenuation spectrum is concerned.

On the other hand, the theoretical model of ultrasonic wave propagation should be provided for the inversion calculation, and the model contains the information of material properties, measurement conditions, and measured ultrasonic spectra; the PSD can be solved with a mathematical equation. By employing a rigorous and fundamental theoretical model, particle sizing with ultrasonic spectra deeply relies on the assumption that the fundamental relationship between ultrasound spectra and requisite physical properties, and ultimately the inversion calculation of the resultant particle size distribution. The accuracy of the retrieval results would be influenced not only by ultrasonic spectra but also by the selection of inversion algorithms. Several inversion algorithms have been investigated and developed in the previous researches to provide an appropriate way to analyze the ultrasonic spectrum and obtain the PSD, for example, the Davidon-Fletcher-Powell (DFP) algorithm, Twomey's method and its improved form $[19,20]$, the optimum regularization technique (ORT) [21], Fruit Fly Optimization (FOA) [22], and the genetic algorithm (GA) [23]. Among them, the ORT is improved by optimizing the regularization factors and can be used in solving the illconditioned equations with a higher stability of solution, and the GA is characterized by its university, robustness, and feasibility for parallel computing. Accordingly, these two inversion algorithms are discussed to determine the effective spectral information.

In the previous investigations, the particle size distribution in suspensions was usually obtained by utilizing the single-mode ultrasonic method extracted from either reflection or transmission signals, which would cause uncertainty to the measurement results due to insufficient information. Starting from analyzing ultrasonic attenuation and phase velocity spectra and comparing the inversed particle size and distribution, a comprehensive measurement method and a carefully designed experimental setup were proposed to provide a noninvasive way in synchronously acquiring these two kinds of ultrasound signals in the present work, which can effectively suppress the interference of different ultrasonic analysis methods in synchronous measurement. In addition, the above two broadband ultrasonic spectra can be synchronously extracted from simultaneous experimental signals, which were implemented by the pulse-echo method, reference reflection method, and through-transmission methods at the same time. Particularly, the relatively novel method of the ultrasonic phase velocity spectrum was also used to inverse PSD with the ORT and GA algorithms. Thus, characteristics of the above various measurement methods were discussed so as to facilitate a suitable understanding on the information content exploitation for characterizing particle size distribution in suspensions using ultrasonic spectra.

\section{Measurement and Analysis Methods of Ultrasonic Spectra}

2.1. Measurement Principles. Figure 1 shows the schematic diagram of the acoustic propagation path with simultaneous measurement of ultrasonic reflection and transmission signals. The measurement apparatus has a geometrically symmetrical structure and consists of two pieces of plexiglass buffer rods (delay line) with the thickness of $l_{1}$, and measurement cell is $l_{2}$. A pair of ultrasonic transducers were mounted on each end of the buffer rods; one can serve as transmitter/receiver (transducer $\mathrm{A}$ ); the other is the receiver with the same parameters (transducer B). In the test run, the compressional wave $A_{0}$ is first generated by ultrasonic transducer $\mathrm{A}$, and it travels along the delay line/sample interface where it is partly reflected and partly transmitted. And then, the reflected wave $A_{1}$ travels back through the delay line and is received by the same transducer. The transmitted portion continues to propagate through the measurement cell and is reflected both on the sample/delay line interface and the delay line/transducer interface; for the same reason, the reflected waves also transmit back and are detected by transducer $\mathrm{A}$, which are termed as $A_{2}$ and $A_{3}$. Finally, transmitted wave $A^{*}$ is received by transducer $\mathrm{B}$. The ultrasonic spectra can be obtained by measuring and analyzing the aforementioned sound signals, and the ultrasonic attenuation coefficient is a measure of the decrease in amplitudes of the 


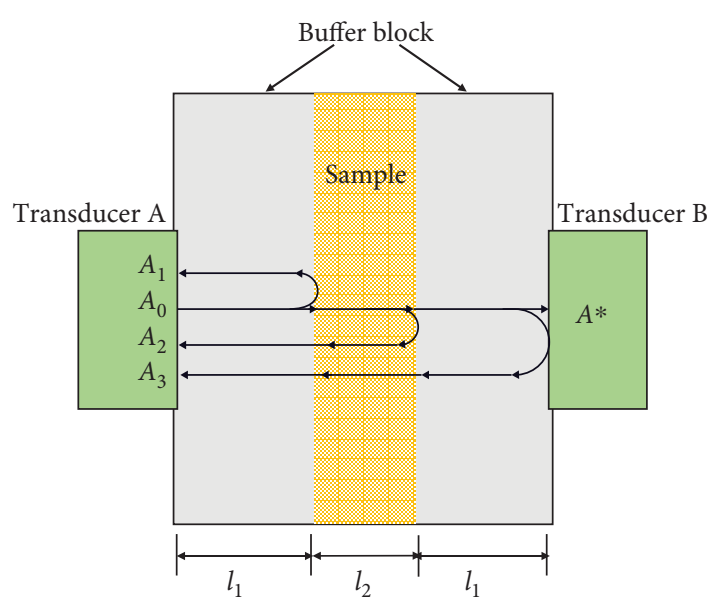

FIGURE 1: Schematic representation of ultrasonic propagation in the measurement cell.

ultrasonic waves (reflection or transmission) per unit distance as they travel through the materials; the velocity is determined by measuring the elapsed time difference and travel distance between any two different signals, which will be discussed in more details below. There is no consideration about the multiple reflections here due to the sharply decaying signals, and a low signal-noise ratio (SNR) often brings a great trouble in separating the effective signals.

2.2. Ultrasonic Attenuation Analysis. In principle, the attenuation coefficient can be calculated by comparing the reduction in the amplitude of the wave traveled across the sample with that of a wave traveled across the buffer rod or a calibration material. The measurement techniques may be operated in a pulse-echo method, reference reflection method, and through-transmission method according to their respective principles.

2.2.1. Pulse-Echo Method. The pulse-echo method [24] only needs one transducer to work; that is, a single transducer is employed to both transmit and receive the ultrasonic signals. This method can provide an absolute measurement by analyzing the sample echoes $A_{1}$ and $A_{2}$, while the latter transmits through the measurement cell and echo $A_{1}$ merely spreads within the buffer rod. The ultrasonic amplitudes are obtained by fast Fourier transform (FFT) with the timedomain signals as

$$
\begin{aligned}
& A_{1}=A_{0} R_{\mathrm{s}} e^{-2 \alpha_{\mathrm{c}} l_{1}}, \\
& A_{2}=A_{0} R_{\mathrm{s}}\left(1-R_{\mathrm{s}}^{2}\right) e^{-2 \alpha_{\mathrm{c}} l_{1}} e^{-2 \alpha_{\mathrm{s}} l_{2}},
\end{aligned}
$$

where $R_{\mathrm{s}}$ is the reflection coefficient of the delay line/sample interface and $\alpha_{\mathrm{c}}$ and $\alpha_{\mathrm{s}}$ represent the attenuation coefficients of the buffer rod and the sample material, with buffer rod length $l_{1}$ and the measurement cell length $l_{2}$, respectively. The ultrasonic attenuation coefficient can be expressed as

$$
\alpha_{\mathrm{s}}=\frac{1}{2} \frac{\ln \left[A_{1}\left(1-R_{\mathrm{s}}^{2}\right) / A_{2}\right]}{l_{2}} .
$$

It is difficult to determine $A_{1}$ and $A_{2}$ directly, but they can be calculated indirectly by using air as the calibration reference, since the total reflection may occur in the delay line/air interface due to the very small acoustic impedance of air compared to that of the buffer rod (e.g., perspex). A group of reflection signals $A_{\mathrm{a}}$ can be measured for the vacant measurement cell, as expressed as follows:

$$
A_{\mathrm{a}}=A_{0} e^{-2 \alpha_{\mathrm{c}} l_{1}}
$$

The reflection coefficient of the delay line/sample interface $R_{\mathrm{s}}$ can be represented by the following equation:

$$
R_{\mathrm{s}}=\frac{A_{1}}{A_{\mathrm{a}}}
$$

It can be seen from equation (4) that the reflection coefficient of the sample is determined by measuring the amplitudes of the pulse echoes reflected from the end of the first buffer rod when the sample and the air are present, respectively. So, the ultrasonic attenuation coefficient of the sample can be calculated as

$$
\alpha_{\mathrm{s}}=\frac{1}{2} \frac{\ln \left[A_{1}\left(1-\left(A_{1} / A_{\mathrm{a}}\right)^{2}\right) / A_{2}\right]}{l_{2}}
$$

Owing to $R_{\mathrm{s}}$ being expressed as the quotient of two pulse amplitudes of $A_{1}$ and $A_{\mathrm{a}}$, the problem of measuring absolute signal amplitudes $A_{0}$ may be avoided, which not only simplifies the expression of the ultrasonic attenuation coefficient but also improves the measurement accuracy.

2.2.2. Reference Reflection Method. The reference reflection method $[25,26]$ belongs to relative measurement, and it utilizes the reflection wave $A_{2 \mathrm{w}}$ for the reference water and reflection wave $A_{2}$ for the sample; they both spread through the measurement cell but possess different amplitudes on account of encountering different materials. This working model is also subjected to pulse-echo measurement with an expression of the ultrasonic attenuation coefficient as

$\alpha_{\mathrm{s}}=\alpha_{\mathrm{w}}+\frac{1}{2} \frac{\ln \left[A_{2 \mathrm{w}}\left(A_{1} / A_{\mathrm{a}}\right)\left(1-\left(A_{1} / A_{\mathrm{a}}\right)^{2}\right) / A_{2} R_{\mathrm{w}}\left(1-R_{\mathrm{w}}{ }^{2}\right)\right]}{l_{2}}$,

where $R_{\mathrm{w}}$ is the reflection coefficient of the delay line/water interface and $\alpha_{\mathrm{w}}$ is the water attenuation coefficient.

It should be noted that the reflection signal $A_{3}$ that transmits through the measurement cell and is then reflected on the delay line/transducer interface is not utilized here, 
because of the interference effects and the sharply attenuated echo signals.

2.2.3. Through-Transmission Method. The throughtransmission method [24] is also a relative measurement in which water transmission wave $A_{1 \mathrm{w}}$ and sample transmission wave $A^{*}$ are adopted. The working model belongs to the pitch-catch principle with two separated transducers employed to transmit and receive the wave signals. The expression of the ultrasonic attenuation coefficient is

$$
\alpha_{\mathrm{s}}=\alpha_{\mathrm{w}}-\frac{\ln \left[A^{*}\left(1-R_{\mathrm{w}}{ }^{2}\right) / A_{1 \mathrm{w}}\left(1-\left(A_{1} / A_{\mathrm{a}}\right)^{2}\right)\right]}{l_{2}} .
$$

It is worth noting that the above three methods are suitable for single-frequency ultrasound processing and can also be used for ultrasonic spectrum analysis. In this case, the spectrum needs to be obtained in advance using the aforementioned FFT technique, and the multifrequency components should be correspondingly calculated with the amplitude at the corresponding frequency, whereby the respective acoustic attenuation spectra can be obtained from the amplitude spectrum analysis.

2.3. Ultrasonic Velocity Analysis. As mentioned earlier, the velocity spectrum is only related to the phase and has nothing to do with the amplitude, so it mainly depends on the time-of-flight (TOF) and the phase difference. Since the pulse-echo method adopts the same testing material, it can hardly be used for the calculation of phase velocity. The through-transmission method is available in principle, but the reference reflection method experiences a sound length twice as far as the former, consequently producing a more distinct phase difference; hence, it is preferable for the reference reflection method in calculating ultrasonic velocity.

When the measurement cell is full of water (as a reference), the time that the ultrasonic wave transmits from the transducer $\mathrm{A}$ and then is reflected is recorded ast $t_{1}$, when the measurement cell is full of sample, for the same propagation process, the corresponding time is represented as $t_{2}$. The propagation time can be treated as

$$
\begin{aligned}
& t_{1}=\frac{2 l_{1}}{c_{\mathrm{c}}}+\frac{2 l_{2}}{c_{\mathrm{w}}}, \\
& t_{2}=\frac{2 l_{1}}{c_{\mathrm{c}}}+\frac{2 l_{2}}{c_{\mathrm{s}}},
\end{aligned}
$$

where $c_{\mathrm{c}}, c_{\mathrm{w}}$, and $c_{\mathrm{s}}$ are the ultrasonic velocities of the buffer rod, water, and sample, respectively ( $c_{\mathrm{w}}$ is known accurately).

It is crucial to firstly calculate propagation time difference $\left(t_{1}-t_{2}\right)$ to solve $c_{\mathrm{s}}$. The formulas are as follows:

$$
\begin{aligned}
c_{\mathrm{s}} & =\frac{c_{\mathrm{w}}}{1-c_{\mathrm{w}}\left(t_{1}-t_{2}\right) / l_{2}}, \\
t_{1}-t_{2} & =\frac{\Delta \mathrm{ps}}{\omega},
\end{aligned}
$$

where $\Delta \mathrm{ps}$ refers to the phase shifts of the two reflection signals and $\omega$ is the angular frequency.

Since the ultrasonic spectra are fundamental physical characteristics and highly rely on the composition and microstructure of the material, they are closely related to particle size and distribution. Thus, it is important to keep the measurement device in good condition to obtain reliable and accurate data. Equally important is the way analysis mode in which different measurement methods serve to obtain the spectral information components and correlations.

\section{Experiments}

3.1. Sample and Experimental Setup. The silicon particle samples (as shown in Figure 2) were employed in the experiments, which could be treated roughly as quasispherical particles. Figure 3 illustrates the particle size distribution of silicon samples measured by the optical image analyzer (model Pip8.1, Omec Inc.), and it denotes a relatively narrow distribution from 7.56 to $15.24 \mu \mathrm{m}$ with the volume median diameter of $11.37 \mu \mathrm{m}$ of the particles. In order to avoid the sedimentation of samples, the experiments should be carried out once after preparing the three silicon-water solutions at volume fractions $8 \%, 10 \%$, and $12 \%$.

The measurement setup configuration and its working process are demonstrated in Figure 4. According to the size range of silicon particles, a pair of ultrasonic longitudinal wave transducers (Panametrics V313-SU, nominal center frequency $5 \mathrm{MHz}$ ) are employed and aligned on both sides of the detection area. Transducer A serves as the transmitter/receiver transducer, working with the ultrasonic pulse signal transmitter/receiver (Panametrics 5800PR, highest excitation frequency $35 \mathrm{MHz}$ ) in the pulse-echo manner, and transducer B is the same model but only takes effect in receiving the ultrasonic signals combined with another ultrasonic pulse signal transmitter/receiver (Panametrics 5077PR, highest excitation frequency $10 \mathrm{MHz}$ ). The measurement apparatus has a geometrically symmetrical structure and consists of two pieces of plexiglass buffer rods with the thickness $10 \mathrm{~mm}$ and measurement cell $6.5 \mathrm{~mm}$, where the buffer rod should be optimized carefully in advance in order to avoid possible confusion due to an echo interference phenomenon. And there are three factors to be considered when selecting appropriately for the optimal experimental results: smoothness, thickness, and material. Firstly, the surface of the buffer rod must have a good smoothness to guarantee that the ultrasonic transducer can be closely aligned with its surface; secondly, the thickness of the buffer rod should separate the reflection signals effectively; and finally, the buffer rod should possess a suitable acoustic impedance to ensure a good signal-to-noise ratio (SNR). One more concern is that the temperature should be carefully kept around $25^{\circ} \mathrm{C}$ by adopting a thermostat bath to avoid the effects of temperature changes on concerning sound propagation. To acquire the data of reflection and transmission signals and analyze the waveform synchronously, a computer with a dual-channel data acquisition card (NI PCI-5114, with a sampling rate of $250 \mathrm{MS} / \mathrm{s}$ ) was employed. 


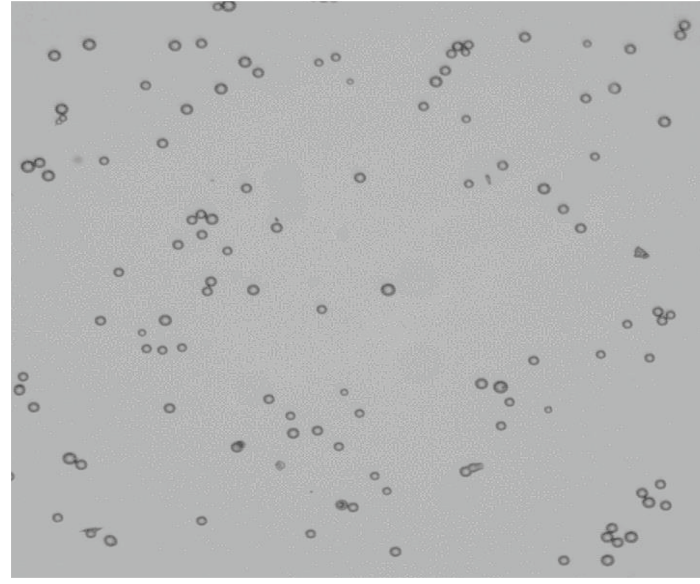

Figure 2: The microscope image of silicon.

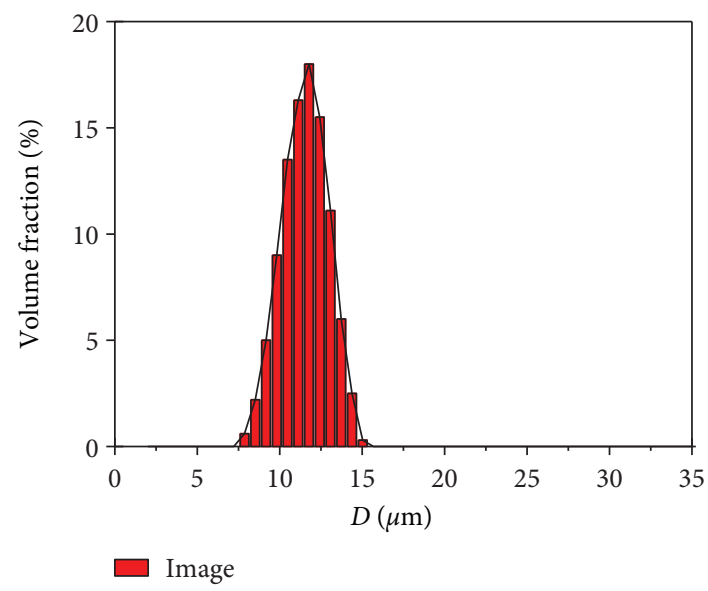

FIgURE 3: The particle size distribution of silicon.

3.2. Experimental Phenomena and Signal Analysis. During testing, the signals were acquired after adjusting the ultrasonic spectrometers: the ultrasonic reflection signals were under the condition of the gain of $40 \mathrm{~dB}$ and transmitting power of $50 \mu \mathrm{J}$ (5800PR), and the transmission signals were under the circumstance of the gain of $20 \mathrm{~dB}$ (5077PR). The experimental reflection and transmission signals of siliconwater solution with the volume fraction $12 \%$ are shown in Figure 5. $A_{0}$ is the initial signal, and $A_{1}, A_{2}$, and $A_{3}$ are the reflection signals of the delay line/sample interface, sample/delay line interface, and delay line/transducer interface, respectively. $A^{*}$ is the transmission signal ultimately reaching the transducer on the other side. It can be seen that $A_{1}$ experiences the shortest distance, $A_{2}$ spreads two more measurement cell length than $A_{1}$, and $A^{*}$ only goes through one more measurement cell length than $A_{1}$, so it is obvious that the phase of $A^{*}$ is behind $A_{1}$ but ahead of $A_{2}$. The reflection signal $A_{3}$ travels the longest distance and has the weaker amplitude compared with other signals, which as mentioned earlier, and is not used here.

The reflection and transmission signals can be applied to analyze the ultrasonic spectra through spectrum analysis based on the fast Fourier transform. The ultrasonic attenuation spectrum related to the signal amplitude can be obtained by using the reflection signals such as $A_{1}$ and $A_{2}$ or the reference signals in the same condition. Similarly, the phase velocity is associated with the unwrapped phase shift, so it is feasible to employ $A_{2}$ to calculate the ultrasonic phase velocity spectrum.

As described before, the ultrasonic spectra can be obtained by using different methods to analyze the reflection and transmission signals. Figure 6 demonstrates the ultrasonic spectra of silicon-water suspensions with different volume fractions; it can be seen from (a), (b), and (c) that the trends of different attenuation spectra are almost consistent and the attenuation grows gradually with the increasing concentration, but the quantities of the through-transmission method are larger than the other two methods (taking silicon-water suspension with $12 \%$ volume fraction as an example, the through-transmission method has an average deviation of $5.2 \%$ from the pulse-echo method and $7.1 \%$ from the reference reflection method). The larger error of through-transmission can be attributed to the working model, which employs two transducers. Although transducer $\mathrm{A}$ and transducer $\mathrm{B}$ are of the same type, the consistency difference and even a slight deviation in installation coaxality could affect the ultrasonic spectra measurements. The phase velocity spectra are displayed in Figure 6(d), which illustrates that the velocity in the suspension is higher than the sound velocity (around $1497 \mathrm{~m} / \mathrm{s}$ ) in water at the same temperature $\left(25^{\circ} \mathrm{C}\right)$, and it also shows an acoustic frequency dispersion that the velocities vary with ultrasonic frequency but without exception grow with the increase of concentration.

\section{Inversion}

For the sake of characterizing particles in suspensions by ultrasonic spectra, first and foremost, the relationship between particle concentration, particle size, and the ultrasonic spectra should be established mathematically. The determination of particle size distribution is not only related to the frequency dependence of experimental ultrasonic spectra but also the theoretical model and the inversion algorithm.

4.1. Theoretical Model. As soon as the ultrasonic attenuation and phase velocity spectra of the suspension have been measured, it is imperative to convert them into a particle size distribution by virtue of an appropriate theory. It is generally based on a mathematical treatment of the physical process that occurs when an ultrasonic wave propagates through the medium. In solid-liquid two-phase flow, the ECAH model is commonly used to model acoustic propagation, which considers the scattering, absorption, and heat effects [27]. The following expression of the complex ultrasonic wave number can be used to interpret the relationship between characteristics of particle and the ultrasonic spectra:

$$
\left(\frac{k}{k_{\mathrm{c}}}\right)^{2}=1+\frac{3 \varphi}{j k_{\mathrm{c}}^{3} R^{3}} \sum_{n=0}^{\infty}(2 n+1) A_{n}
$$




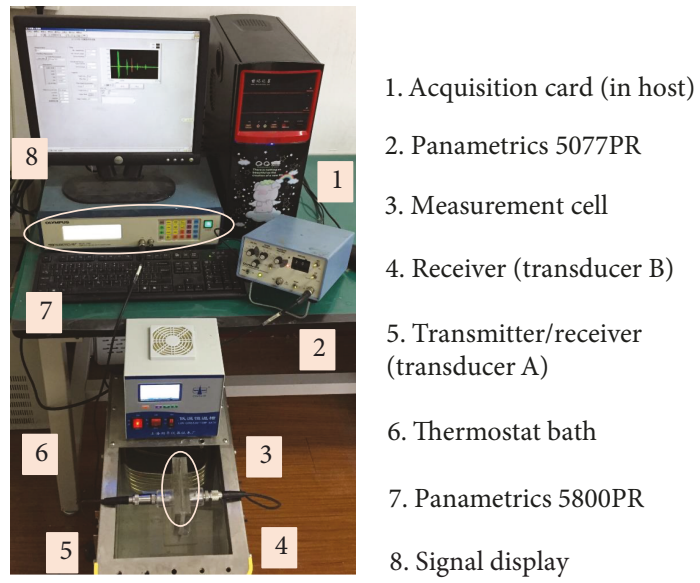

(a)

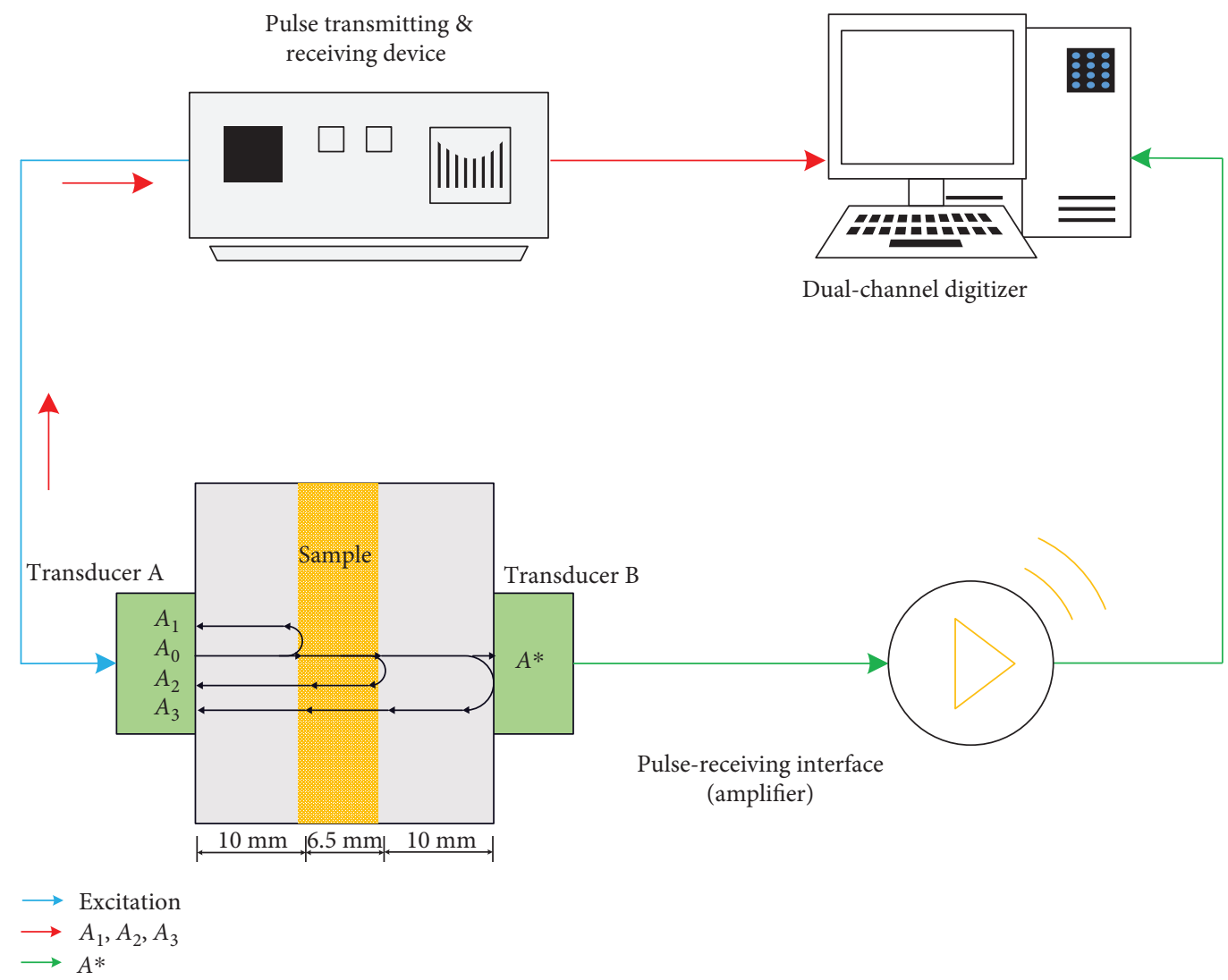

(b)

Figure 4: (a) Photo of the measurement setup configuration. (b) Sketch of the ultrasonic spectrometer used to measure the ultrasonic properties of silicon aqueous suspensions.

with

$$
k=\frac{\omega}{c_{\mathrm{s}}(\omega)}+j \alpha_{\mathrm{s}}(\omega),
$$

where $\alpha_{\mathrm{s}}$ and $c_{\mathrm{s}}$ refer to the sample attenuation coefficient and phase velocity, respectively, which are associated with the ultrasonic frequency in the two-phase measurement system; $k_{\mathrm{c}}=2 \pi / \lambda$ is the compress wave number; $j=\sqrt{-1} \cdot \varphi$ refers to

the volume fraction of particles; $R$ is the radius of the particles; $n$ denotes the scattered level; and $A_{n}$ is known as the scattering coefficient, reflecting the interaction of ultrasonic waves and suspended particles in media; it can be solved by combining the compression wave function and the boundary conditions.

It should be noted that particles are always in a polydisperse condition, so the corresponding attenuation coefficient and velocity should be evaluated from the superposition of contribution from every single bin of particles and can be 


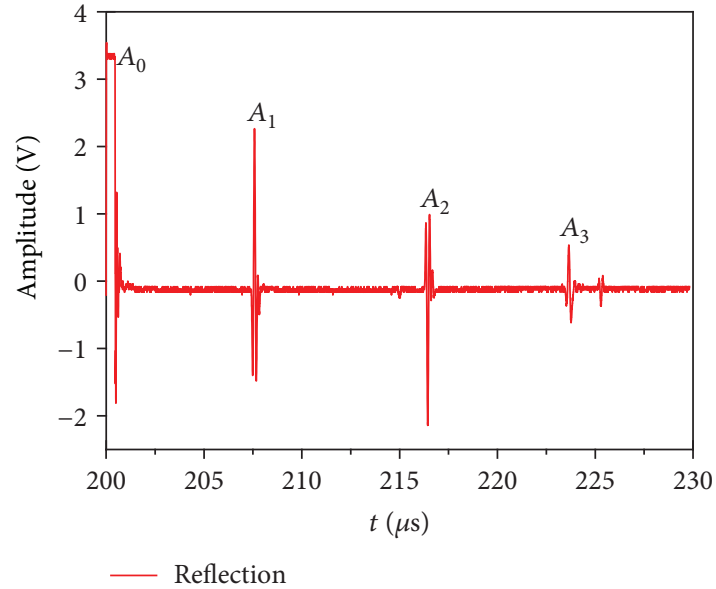

(a)

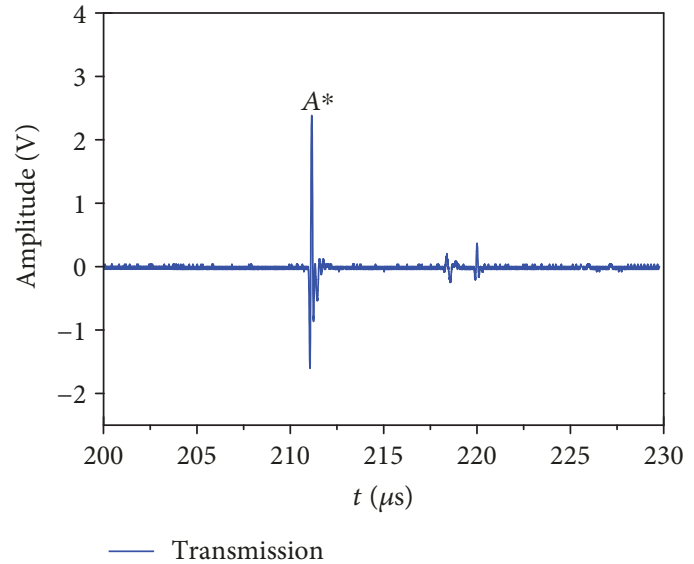

(b)

FIgURE 5: Time-domain signals of ultrasound for samples: (a) reflection signals and (b) transmission signals.

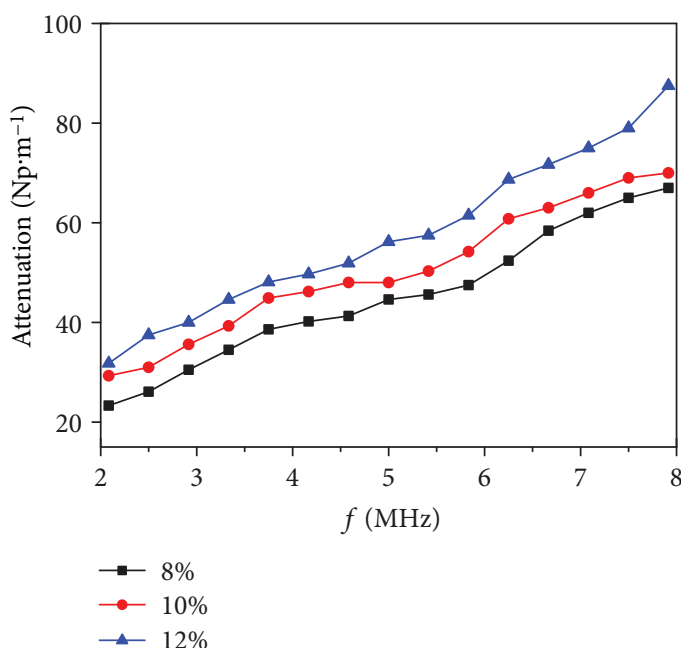

(a)

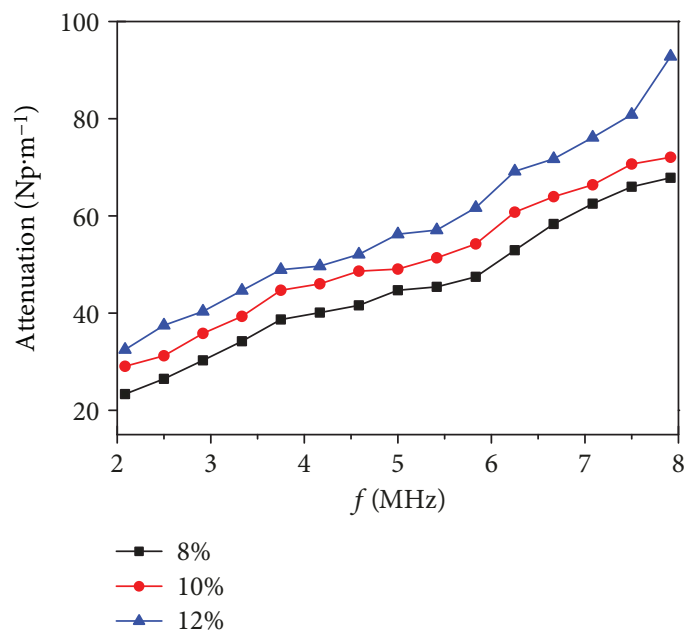

(c)

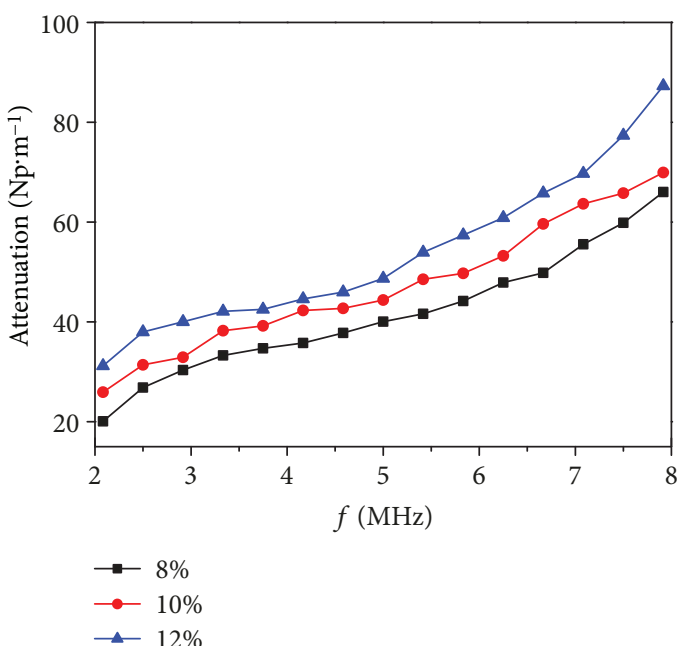

(b)

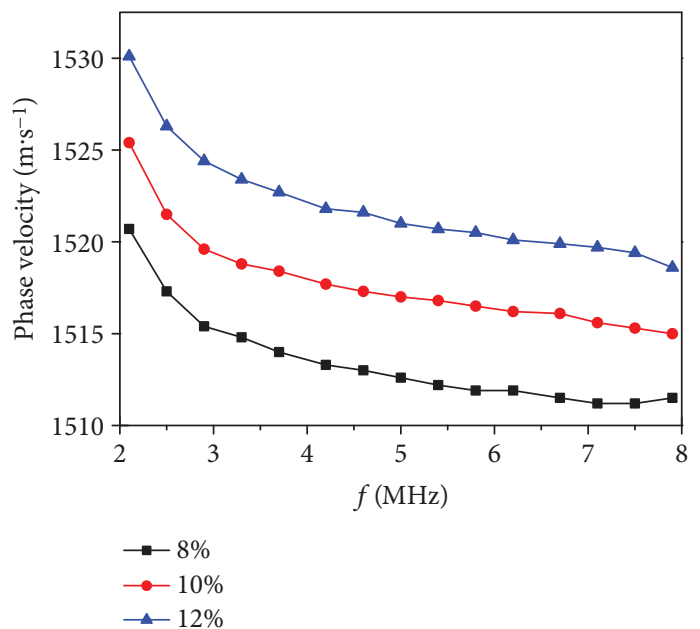

(d)

FIGURE 6: Ultrasonic spectra obtained by different measurement methods: (a) attenuation spectra with the pulse-echo method, (b) attenuation spectra with the reference reflection method, (c) attenuation spectra with the through-transmission method, and (d) phase velocity spectra with the reference reflection method. 
expressed as follows:

$$
\begin{array}{r}
\alpha_{\mathrm{s}}=\frac{3 \varphi}{2 k_{\mathrm{c}}^{2}} \sum_{j=1}^{N} \frac{q_{j}}{R_{j}^{3}} \sum_{n=0}^{\infty}(2 n+1) \operatorname{Re}\left(A_{n}\left(R_{j}, \omega\right)\right), \\
\frac{1}{c_{\mathrm{s}}}-\frac{1}{c_{\mathrm{w}}}=\frac{3 \varphi}{2 \omega k^{2}} \sum_{j=1}^{N} \frac{q_{j}}{R_{j}^{3}} \sum_{n=0}^{\infty}(2 n+1) \operatorname{Im}\left(A_{n}\left(R_{j}, \omega\right)\right),
\end{array}
$$

where $c_{\mathrm{w}}$ is phase velocity of water, which is usually known; $\rho$ is the equivalent density of suspensions; $q_{j}$ is the volume fraction when the particle radius ranges from $R_{j}$ to $R_{j+1}$; Re is the operation of taking the real part of a complex array; and Im is for the imaginary part operation.

The classical ECAH model is relatively comprehensive and requires numerous material parameters as shown in Table 1 [28]. If there is no need to realize real-time analysis, the coefficient matrix can be calculated first to inverse the particle size distribution.

4.2. Inversion Algorithm. The principle of PSD measurement is based on the properties of ultrasonic spectra generated by different particle sizes and is determined by seeking the PSD which gives the best fit between theory and experimental data. The inversion algorithm for particle size can be divided into two types: the dependent model and independent model. For the former algorithm in inversion problems, it is usually assumed that the PSD follows a certain function form such as Rosin-Rammler distribution (R-R), normal distribution, and log-normal distribution. For example, the expression of R-R distribution function can be written as

$$
\frac{d V}{d R}=\frac{k}{\bar{R}}\left(\frac{R}{\bar{R}}\right)^{k-1} \exp \left[-\left(\frac{R}{\bar{R}}\right)^{k}\right] \text {, }
$$

where $d V / d R$ is the frequency distribution of particle volume; $\bar{R}$ is the characteristic radius; and $k$ is the distribution parameter, namely, the narrowness index of the distribution, which is a dimensionless quantity.

The root mean square error can describe the difference between the simulated and the measured spectra as

$$
E_{\mathrm{RMS}}=\sqrt{\frac{1}{N} \sum_{j=1}^{N}\left(\frac{\alpha_{\mathrm{s}}-\alpha_{\mathrm{m}}}{\alpha_{\mathrm{m}}}\right)^{2}},
$$

where $\alpha_{\mathrm{m}}$ is the measured spectrum and $\alpha_{\mathrm{s}}$ is the simulated one. The optimization process can be carried out until the computer terminates the search when the objective function $E_{\mathrm{RMS}}$ reaches the minimum value, and then the optimal characteristic of $\bar{R}$ and $k$ can be determined.

As a global optimization algorithm, the genetic algorithm is suitable for a dependent model, and it is a valid optimization approach based on the natural selection and the theory of genetics, searching global optimization solution without any initialization information [23]. Thus, the authors have developed an inversion algorithm based on the genetic algorithm before, where some crucial parameters, e.g., maximum generations, population size, and genetic operators (crossover fraction and mutation fraction), were optimized for particle size characterization with its improved form (see [25]). This method has already demonstrated some advantages compared with other local optimization methods because of global convergence, great robustness, suitable for parallel computing, and so on.

At the same time, an optimum regularization technique has been proposed, as an independent model algorithm, which is improved by optimizing the regularization factors combined with mathematic operations such as Generalized Cross Validation [29], to solve the ill-conditioned equations and yield a higher stability of solution. For this purpose, the major equations (12) and (13) must be modified by altering calculation of coefficient matrix and the form of input ultrasonic spectra in order to obtain more accurate particle size distribution; hence, the two equations are converted into linear equations $A F=G$ after discretization as follows:

$$
\begin{aligned}
& \underbrace{\frac{3 \varphi}{2} \cdot \int_{\Delta R} \frac{1}{k_{c}^{2} R_{j}^{3}} \sum_{n=0}^{\infty}(2 n+1) \operatorname{Re}\left(A_{n}\left(R_{j}, \omega_{i}\right)\right) \cdot d R}_{A_{i, j}} \cdot \underbrace{\sum V\left(\Delta R_{i}\right)}_{F_{i}}=\underbrace{\alpha_{s}\left(\omega_{i}\right)}_{G_{i}}, \\
& \underbrace{\frac{3 \varphi}{2} \cdot \int_{\Delta R} \frac{1}{k_{c}^{2} \omega_{i} R_{j}^{3}} \sum_{n=0}^{\infty}(2 n+1) \operatorname{Im}\left(A_{n}\left(R_{j}, \omega_{i}\right)\right) \cdot d R}_{A_{i, j}} \cdot \underbrace{\sum V\left(\Delta R_{j}\right)}_{F_{j}}=\underbrace{\frac{1}{c_{s}\left(\omega_{i}\right)}-\frac{1}{c_{w}}}_{G_{i}},
\end{aligned}
$$

where $A$ is the coefficient matrix, $F$ is the discrete volume distribution of particle size to be determined, and $G$ is the vector consisting of experimental ultrasonic attenuation or the phase velocity spectrum at different frequencies, while the subscripts $i$ and $j$ indicate the number of discrete classifications of the radius for particles and frequency for ultrasonic attenuation or phase velocity, respectively. 
TABle 1: Physical parameters of water and silicon.

\begin{tabular}{lccccccc}
\hline & $\rho\left(\mathrm{kg} \cdot \mathrm{m}^{-3}\right)$ & $c\left(\mathrm{~m} \cdot \mathrm{s}^{-1}\right)$ & $\alpha\left(\mathrm{Np} \cdot \mathrm{m}^{-1}\right)$ & $C_{\mathrm{p}}\left(\mathrm{J} \cdot \mathrm{kg}^{-1} \cdot \mathrm{K}\right)$ & $\tau\left(\mathrm{W} \cdot \mathrm{m}^{-1} \cdot \mathrm{K}\right)$ & $\beta\left(\mathrm{K}^{-1}\right)$ & $\eta\left(\mathrm{N} \cdot \mathrm{m}^{-2}\right)$ \\
\hline Water & 997 & 1483 & $2.53 \times 10^{-14} f^{2}$ & 4178.5 & 0.595 & $2.57 \times 10^{-4}$ & - \\
Silicon & 2185 & 5968 & $2.6 \times 10^{-22} f^{2}$ & 729 & 1.6 & $1.35 \times 10^{-6}$ & $3.09 \times 10^{10}$ \\
\hline
\end{tabular}

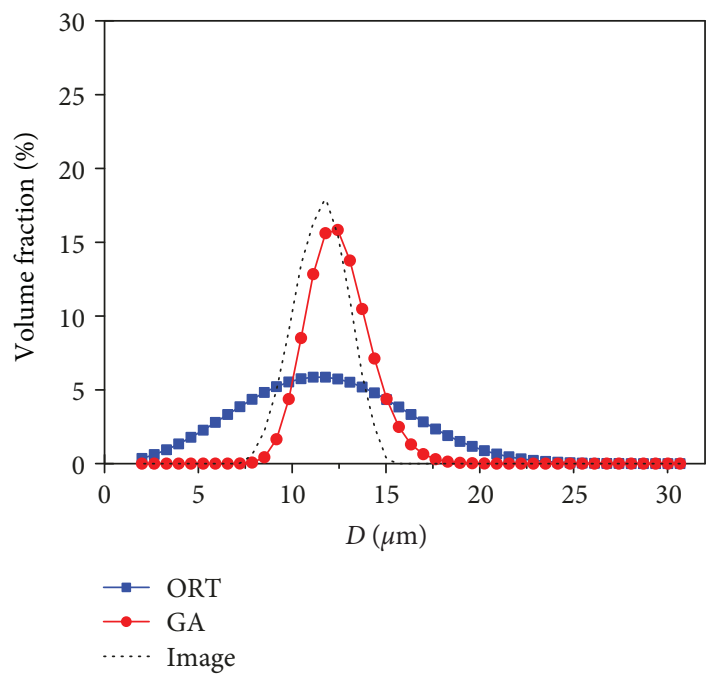

(a)

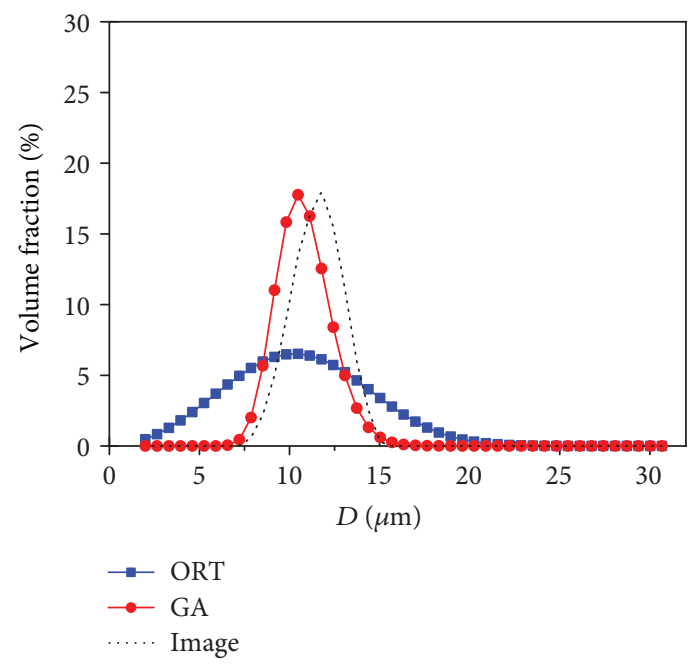

(c)

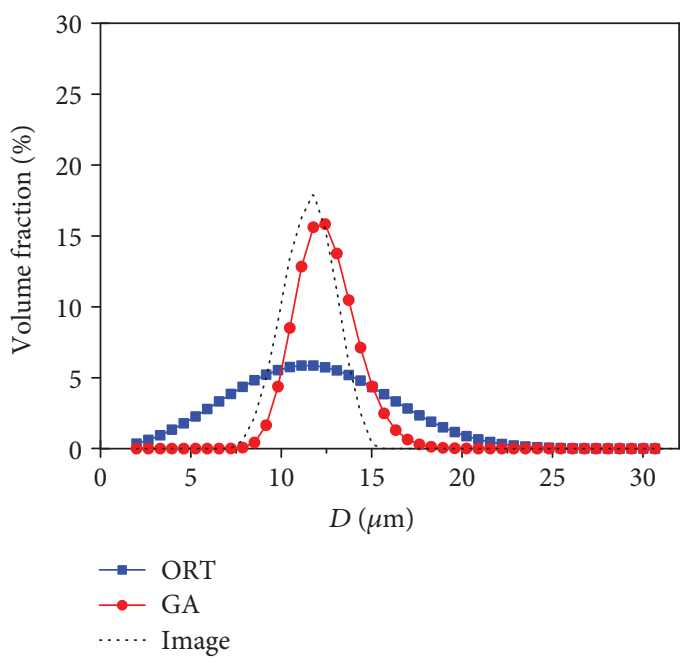

(b)

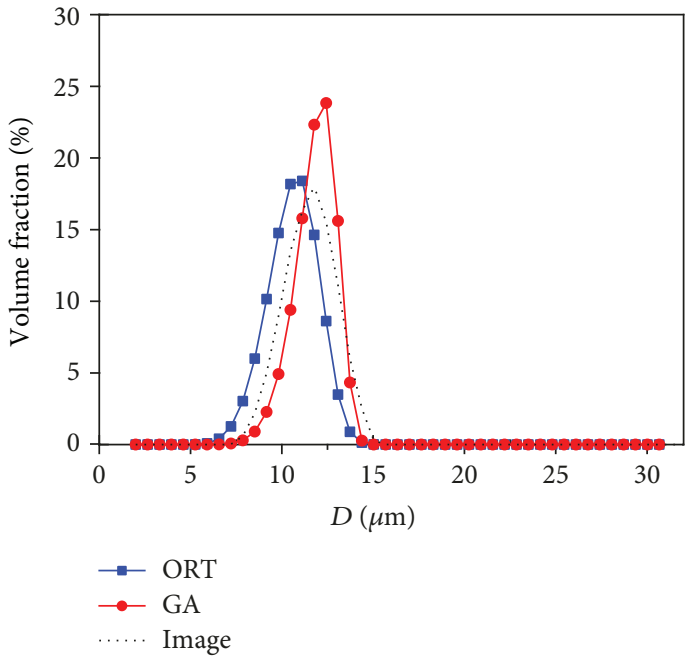

(d)

FIGURE 7: Particle size distribution with different ultrasonic spectra: (a) attenuation spectra with the pulse-echo method, (b) attenuation spectra with the reference reflection method, (c) attenuation spectra with the through-transmission method, and (d) phase velocity spectra with the reference reflection method.

\section{Results and Discussions}

The ultrasonic attenuation/velocity spectra at the volume fraction of $12 \%$ silicon-water suspension were adopted to calculate the particle size distribution of samples. During the calculations, the spectra measured by different methods were analyzed with different inversion algorithms. Figure 7 illustrates the PSDs of the sample obtained from different ultrasonic spectra; (a), (b), and (c) are inversed by attenuation spectra with different measurement methods, which show a good agreement in the trend and distribution width with the same inversion algorithm. In addition, the corresponding volume median diameters $\left(D_{\mathrm{v} 50}\right)$ and distribution width of the sample are shown in Table 2; it can be seen that the results obtained by the pulse-echo method are much closer to the reference reflection method than the throughtransmission method, and their volume median diameters have a relative deviation less than $2 \%$, while the throughtransmission method deviates from the other two methods, yielding an error still less than $15 \%$. As described in the 
TABLE 2: Comparison of the sample volume median diameter measured by ultrasonic spectra and image analysis (unit: $\mu \mathrm{m}$ ).

\begin{tabular}{lccccccc}
\hline $\begin{array}{l}\text { Deviation } \\
\text { Approach }\end{array}$ & \multirow{2}{*}{ Method } & \multicolumn{3}{c}{$\begin{array}{c}\text { Pulse-echo } \\
\left(D_{\mathrm{v} 90}-D_{\mathrm{v} 10}\right) / D_{\mathrm{v} 50}\end{array}$} & $D_{\mathrm{v} 50}$ & $\left(D_{\mathrm{v} 90}-D_{\mathrm{v} 10}\right) / D_{\mathrm{v} 50}$ & \multicolumn{2}{c}{ Through-transmission } \\
$D_{\mathrm{v} 50}$ & $\left(D_{\mathrm{v} 90}-D_{\mathrm{v} 10}\right) / D_{\mathrm{v} 50}$ \\
\hline \multirow{2}{*}{ Attenuation } & ORT & $11.54(+1.5 \%)$ & 0.98 & $11.43(+0.5 \%)$ & 1.04 & $10.56(-7.1 \%)$ \\
& $\mathrm{GA}$ & $12.36(+8.7 \%)$ & 0.41 & $12.21(+7.3 \%)$ & 0.40 & $10.58(-6.9 \%)$ & 0.52 \\
\multirow{2}{*}{ Phase velocity } & ORT & $/$ & $/$ & $10.27(-9.7 \%)$ & 0.42 & $/$ & $/$ \\
Image & GA & $/$ & $/$ & $11.62(+2.2 \%)$ & 0.29 & 1 & 1 \\
\hline
\end{tabular}

context, the amplitude of ultrasonic attenuation spectra with the through-transmission makes a slight difference from those of other two methods, mainly causing the deviation of volume median diameters.

Figure $7(d)$ illustrates the particle size distribution generated by the ultrasonic phase velocity spectra that also well reflect the particle size information, and it can obviously reflect that the width of size distribution is narrower compared with those of ultrasonic attenuation spectra, while the volume median diameter is comparative. It reveals that the widths of particle size distributions obtained by the phase velocity spectra method are relatively close to the image method compared with the attenuation spectra method whenever the inversion algorithms are chosen.

Regarding the inversion algorithm, both GA and ORT algorithms can recover relatively reasonable particle size distribution, including the successful findings of the median value and width of the particle system. As shown in Table 2, the volume median diameters inversed by GA and ORT with the same measurement methods have a deviation from image analysis less than $10 \%$. For the inversion of the attenuation spectrum, the ORT algorithm gives a wider particle size distribution compared with GA algorithm, and the latter is close to the result of the image method. But in the case of phase velocity spectra, it shows that the width of particle size distribution obtained by the ORT algorithm is nearly the same as that of the image method and only slightly wider than that of the GA algorithm. It depicts an inconsistent adaptability to different ultrasonic spectra for the ORT algorithm.

If the influence of spectral information is merely considered, it can be found that there is an obvious relative variation for the attenuation spectrum itself (see Figure 6), which is usually advantageous for analyzing the particle size distribution. However, through further analysis of the coefficient matrix (sensitive matrix), it can be seen that not only the spectral information but also the involved mathematical problems affect results. For instance, the condition numbers of the matrix after the singular value decomposition for the attenuation spectrum solution are particularly larger (up to $3 \times 10^{9}$ ), about ten times of those for the phase velocity. Hence, it is likely to encounter a more serious illconditioned problem. This can help to explain that the inversed PSD of the attenuation spectrum is often too wide with ORT. However, the performance of the GA algorithm seems to be more stable on the whole; no matter what attenuation spectra or phase velocity spectra are chosen, there is no great deviation from the image analysis. As the GA algorithm here belongs to the dependent model, the calculation of particle size distribution must obey the Rosin-Rammler distribution function, which in turn exerts a strong constraint for inversion. While for the ORT algorithm (independent model), there is no need for such a constraint of distribution function.

Back to the measurement method itself, the frequencydependent ultrasonic spectra of the sample are measured in a basic similar fashion as mentioned in these three methods, and the analysis procedures are automated and straightforward, while the differences and considerations in transducer installation, methodology principle, and signal analysis need to be stressed again. Firstly, the pulse-echo method and reference reflection method use a single transducer to both transmit and receive the ultrasonic waves while the throughtransmission method employs a pair of transducers to work. Given that case, it is obvious that the measurement device of the two former methods is relatively simple and commercially available regardless of caring for the transducer installation coaxality. Secondly, the reference reflection method and the through-transmission method belong to relative measurement; however, the pulse-echo method is an absolute measurement, which means that the pulse-echo method is easier to operate without considering the calibration of reference material. Finally, the pulse-echo method and reference reflection method must adopt the reflection signals during spectral calculation, so it is important to select the proper buffer rod and bring a reliable impedance difference for accurate reflection signal acquisition, which need to be considered carefully in the practical applications.

\section{Conclusions}

The experimental setup was specially designed to provide a noninvasive way in synchronously acquiring ultrasonic reflection and transmission signals, which can be utilized to measure the frequency-dependent ultrasonic properties of suspensions. To obtain the particle size distribution, the pulse-echo method, reference reflection method, and through-transmission method were employed to extract the ultrasonic attenuation and phase velocity spectra combined with the inversion algorithms GA and ORT. The particle size distributions inversed by these two inversion algorithms both well reflect the particle information and bring a relative deviation less than $10 \%$ about the volume median diameter with 
image analysis, which indicates that different methods have a high stability and reliability.

In brief, the reference reflection method is easy to obtain the attenuation and velocity spectra at the same time. However, as far as the results discussed in the present paper are concerned, the procedure of inversion needs to pay more attention to the adaptability of different algorithms to various spectra, which often influences the width of particle size distribution rather than the median diameter. Further, considering the online measurement problem, the pulse-echo method is very advantageous for the design of the concise probe since only one transducer is used, and the reference material can be avoided during the absolute measurement. Similarly, the through-transmission method is ideal for mounting a pair of transducers on the tube wall to implement a real nondestructive testing, which is necessary in many process measurements.

\section{Data Availability}

The data used to support the findings of this study are available from the corresponding author upon request.

\section{Conflicts of Interest}

The authors declare that there is no conflict of interest regarding the publication of this paper.

\section{Acknowledgments}

The present work is supported by the National Natural Science Foundation of China (NSFC 51776129 and 51676130), which is gratefully acknowledged.

\section{References}

[1] N. Samet, P. Maréchal, and H. Duflo, "Ultrasonic characterization of a fluid layer using a broadband transducer," Ultrasonics, vol. 52, no. 3, pp. 427-434, 2012.

[2] F. Babick, F. Hinze, and S. Ripperger, "Dependence of ultrasonic attenuation on the material properties," Colloids and Surfaces A: Physicochemical and Engineering Aspects, vol. 172, no. 1-3, pp. 33-46, 2000.

[3] A. Richter, T. Voigt, and S. Ripperger, "Ultrasonic attenuation spectroscopy of emulsions with droplet sizes greater than 10 $\mu \mathrm{m}$," Journal of Colloid and Interface Science, vol. 315, no. 2, pp. 482-492, 2007.

[4] M. J. W. Povey, "Ultrasound particle sizing: a review," Particuology, vol. 11, no. 2, pp. 135-147, 2013.

[5] X. Z. Wang, L. Liu, R. F. Li et al., "Online characterisation of nanoparticle suspensions using dynamic light scattering, ultrasound spectroscopy and process tomography," Chemical Engineering Research and Design, vol. 87, no. 6, pp. 874-884, 2009.

[6] A. Shukla, A. Prakash, and S. Rohani, "Online measurement of particle size distribution during crystallization using ultrasonic spectroscopy," Chemical Engineering Science, vol. 65, no. 10, pp. 3072-3079, 2010.

[7] M. Su, M. Xue, X. Cai, Z. Shang, and F. Xu, "Particle size characterization by ultrasonic attenuation spectra," Particuology, vol. 6, no. 4, pp. 276-281, 2008.
[8] O. L. Alves, A. C. M. D. Moraes, M. B. Simões et al., "Measurement and characterization of particles by acoustic methods, part 1: concepts and procedures in ultrasonic attenuation spectroscopy," Nanotoxicology, 2014, BS ISO 20998-1.

[9] H. S. Hou, "A study on measuring nanoparticle size distribution based on ultrasonic attenuation spectrum," Acta Acustica, vol. 35, no. 5, pp. 508-514, 2010.

[10] M. Xue, M. Su, L. Dong, Z. Shang, and X. Cai, “An investigation on characterizing dense coal-water slurry with ultrasound: theoretical and experimental method," Chemical Engineering Communications, vol. 197, no. 2, pp. 169-179, 2009.

[11] W. Zhou, M. Su, and X. Cai, “Advances in nanoparticle sizing in suspensions: dynamic light scattering and ultrasonic attenuation spectroscopy," Kona Powder and Particle Journal, vol. 34, pp. 168-182, 2017.

[12] J. Gu, M. Su, and X. Cai, "In-line measurement of pulverized coal concentration and size in pneumatic pipelines using dual-frequency ultrasound," Applied Acoustics, vol. 138, no. 10, pp. 163-170, 2018.

[13] P. Mougin, D. Wilkinson, K. J. Roberts, and R. Tweedie, "Characterization of particle size and its distribution during the crystallization of organic fine chemical products as measured in situ using ultrasonic attenuation spectroscopy," The Journal of the Acoustical Society of America, vol. 109, no. 1, pp. 274-282, 2001.

[14] J. Gu, H. Yang, F. Fan, and M. Su, "A transmission and reflection coupled ultrasonic process tomography based on cylindrical miniaturized transducers using PVDF films," Journal of Instrumentation, vol. 12, no. 12, article 12026, 2017.

[15] J. Wu, "Experimental study on multi-parameters of particle two-phase system based on ultrasonic measurements," Journal of Engineering Thermophysics, vol. 33, no. 9, pp. 1531-1534, 2012.

[16] U. Riebel and F. Loffler, "The fundamentals of particle size analysis by means of ultrasonic spectrometry," Particle and Particle Systems Characterization, vol. 6, no. 1-4, pp. 135143, 1989.

[17] M. S. Hughes, A. L. Klibanov, J. N. Marsh, J. G. Miller, and G. H. Brandenburger, "Broadband time-domain reflectometry measurement of attenuation and phase velocity in highly attenuating suspensions with application to the ultrasound contrast medium Albunex ${ }^{\circledR}$," The Journal of the Acoustical Society of America, vol. 108, no. 2, pp. 813-820, 2000.

[18] X. Ji, M. Su, J. Chen, X. Wang, and X. Cai, "A novel method for plastic particle sizing in suspension based on acoustic impedance spectrum," Ultrasonics, vol. 77, no. 5, pp. 224-230, 2017.

[19] T. Igushi and H. Yoshida, "Investigation of low-angle laser light scattering patterns using the modified Twomey iterative method for particle sizing," Review of Scientific Instruments, vol. 82, no. 1, pp. 2-4, 2011.

[20] J. Zhou, F. Moshary, B. Gross, and S. Ahmed, "Particle size and refractive index retrieval from the backscattering spectrum of white light using the Twomey iterative method: simulation and experiment," Applied Optics, vol. 45, no. 26, pp. $6876-$ 6885, 2006.

[21] H. Schnablegger and O. Glatter, "Optical sizing of small colloidal particles: an optimized regularization technique," Applied Optics, vol. 30, no. 33, pp. 4889-4896, 1991.

[22] Z. He, H. Qi, Y. Yao, and L. Ruan, "Inverse estimation of the particle size distribution using the fruit fly optimization 
algorithm," Applied Thermal Engineering, vol. 88, no. 15, pp. 306-314, 2015.

[23] E. Assareh, M. A. Behrang, M. R. Assari, and A. Ghanbarzadeh, "Application of PSO (particle swarm optimization) and GA (genetic algorithm) techniques on demand estimation of oil in Iran," Energy, vol. 35, no. 12, pp. 52235229, 2010.

[24] D. J. McClements and P. Fairley, "Ultrasonic pulse echo reflectometer," Ultrasonics, vol. 29, no. 1, pp. 58-62, 1991.

[25] H. Yang, M. Su, X. Wang, J. Gu, and X. Cai, "Particle sizing with improved genetic algorithm by ultrasound attenuation spectroscopy," Powder Technology, vol. 304, no. 18, pp. 2026, 2016.

[26] D. J. McClements and P. Fairley, "Frequency scanning ultrasonic pulse echo reflectometer," Ultrasonics, vol. 30, no. 6, pp. 403-405, 1992.

[27] G. E. Eliçabe and L. H. García-Rubio, "Latex particle size distribution from turbidimetry using inversion techniques," Journal of Colloid and Interface Science, vol. 129, no. 1, pp. 192200, 1989.

[28] S. Lemlikchi, M. Asmani, H. Djelouah, and P. Schaaf, "Direct transduction method for measuring the ultrasonic attenuation in $\mathrm{Si}(111)$ in the frequency range $100 \mathrm{MHz}-1 \mathrm{GHz}$," Measurement, vol. 100, no. 6, pp. 279-287, 2017.

[29] M. Su, F. Xu, X. Cai, K. Ren, and J. Shen, “Optimization of regularization parameter of inversion in particle sizing using light extinction method," China Particuology, vol. 5, no. 4, pp. 295299, 2007. 


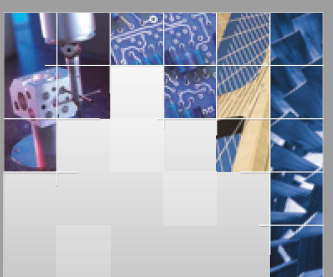

\section{Enfincering}
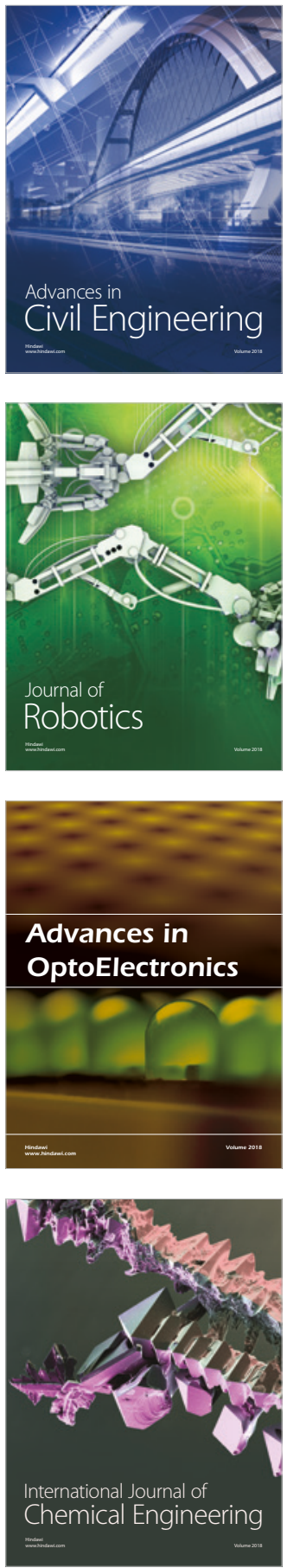

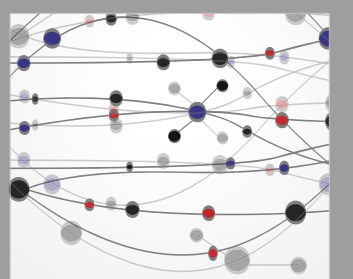

\section{Rotating \\ Machinery}

The Scientific World Journal

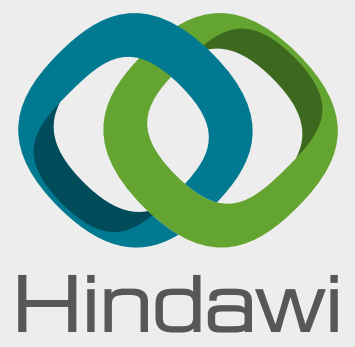

Submit your manuscripts at

www.hindawi.com
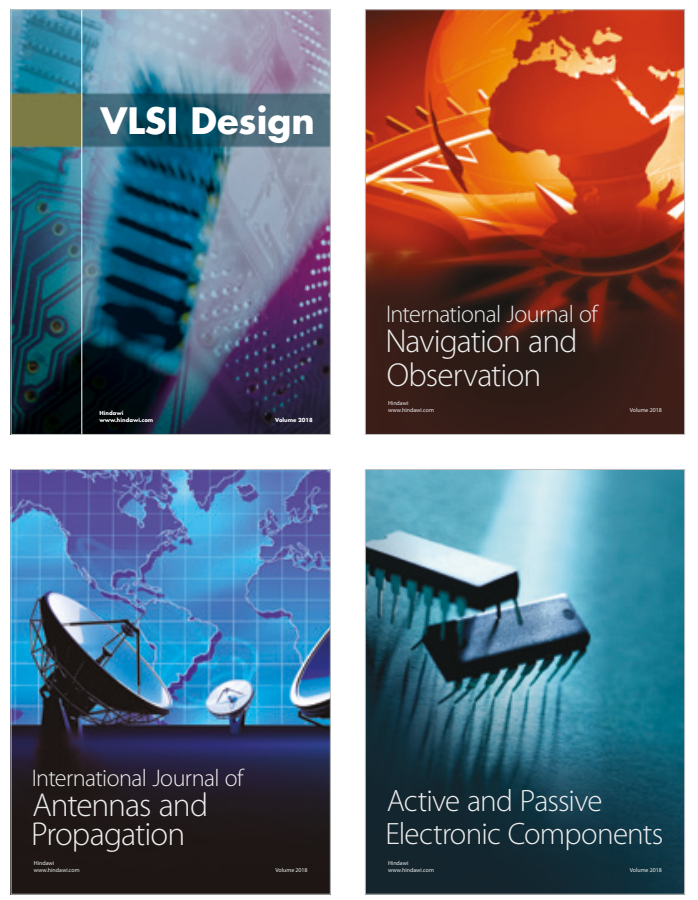
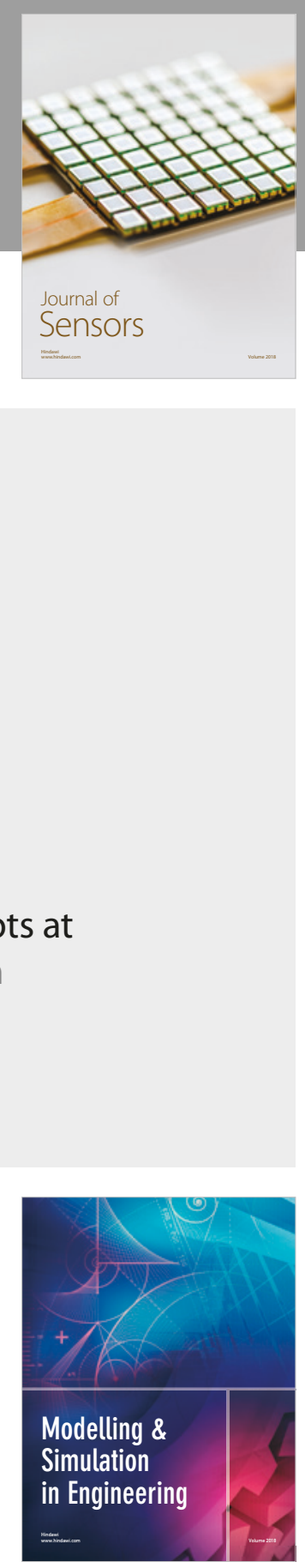

\section{Advances \\ Multimedia}
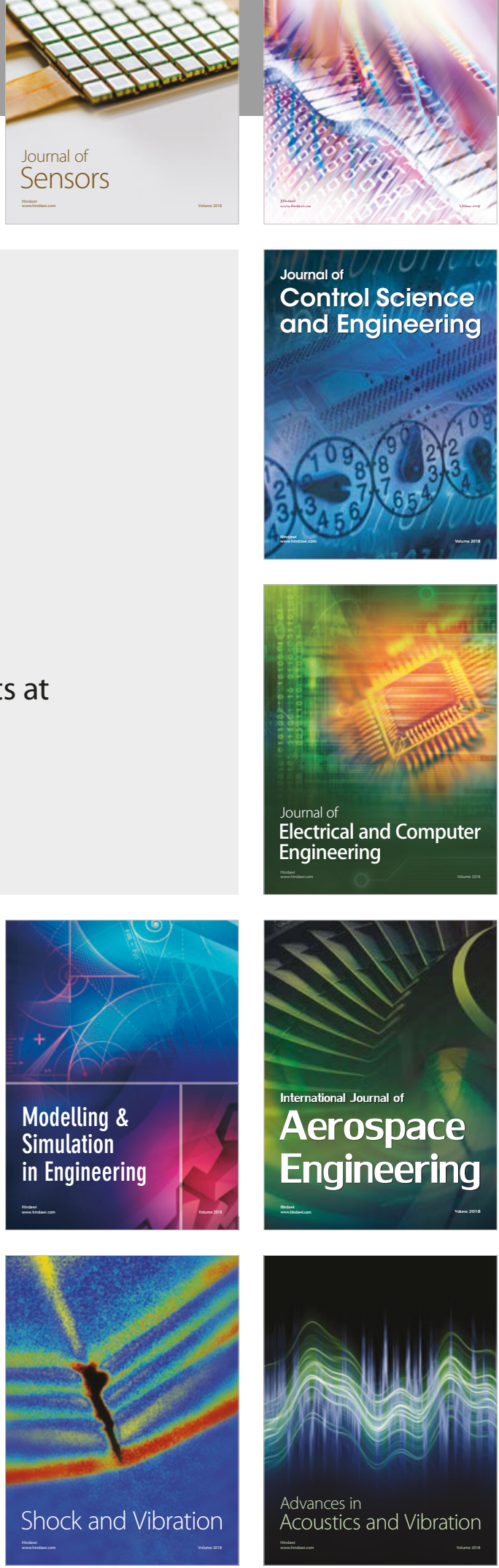